\title{
Conditional disruption of $\mathrm{I} \kappa \mathrm{B}$ kinase 2 fails to prevent obesity-induced insulin resistance
}

\author{
Mathias Röhl, ${ }^{1}$ Manolis Pasparakis, ${ }^{2}$ Stephanie Baudler,,3 Julia Baumgartl, 1,3 \\ Dinesh Gautam, ${ }^{1}$ Marion Huth, ${ }^{2}$ Rossana De Lorenzi, ${ }^{2}$ Wilhelm Krone, ${ }^{1}$ \\ Klaus Rajewsky, ${ }^{4}$ and Jens C. Brüning ${ }^{1,3}$ \\ ${ }^{1}$ Klinik II und Poliklinik für Innere Medizin der Universität zu Köln and Center for Molecular Medicine Cologne (CMMC), \\ Cologne, Germany \\ ${ }^{2}$ European Molecular Biology Laboratory Mouse Biology Programme, Monterotondo, Italy \\ ${ }^{3}$ Institute for Genetics, University of Cologne and CMMC, Cologne, Germany \\ ${ }^{4}$ Center for Blood Research, Harvard Medical School, Boston, Massachusetts, USA
}

\begin{abstract}
The inhibitor of NF-KB (IKB) kinases (IKK1 $[\alpha]$ and IKK2 $[\beta])$, the catalytic subunits of the IKK complex, phosphorylate $\mathrm{I} \kappa \mathrm{B}$ proteins on serine residues, targeting them for degradation and thus activating the transcription factor NF- $\mathrm{KB}$. More recently, IKK2 has been implicated in mediation of insulin resistance caused by obesity, lipid infusion, and TNF- $\alpha$ stimulation, since salicylate and aspirin, known inhibitors of IKK activity, can reverse insulin resistance in obese mouse models. To further genetically elucidate the role of IKK2 in obesity-mediated insulin resistance, we have conditionally inactivated the mouse IKK2 gene in adult myocytes by Cre-loxP-mediated recombination in vivo. We have investigated the development of obesity-induced insulin resistance in muscle-specific IKK2 knockout mice and mice exhibiting a 50\% reduction of IKK2 expression in every tissue and have found that, after gold thioglucose treatment, wild-type and mutant mice developed obesity to a similar extent. Surprisingly, no difference in obesity-induced insulin resistance was detectable, either at a physiological or at a molecular level. Moreover, impaired glucose tolerance resulting from a high-fat diet occurred to the same degree in control and IKK2 mutant mice. These data argue against a substantial role for muscular IKK2 in mediating obesity-induced insulin resistance in these models in vivo.
\end{abstract}

J. Clin. Invest. 113:474-481 (2004). doi:10.1172/JCI200418712.

\section{Introduction}

$\mathrm{NF}-\mathrm{KB}$ plays a key role in inflammatory and immune responses (1). In unstimulated cells, NF- $\mathrm{KB}$ dimers are bound to inhibitory proteins, the I $\kappa \mathrm{Bs}$, and thereby retained in the cytoplasm. In response to cell stimulation, mainly by proinflammatory cytokines such as TNF- $\alpha$, a multisubunit protein kinase, the IKB kinase (IKK), is activated (2). IKK then phosphorylates two serine residues in the $\mathrm{N}$-termini of the IKBs, thereby targeting $\mathrm{I} \kappa \mathrm{B}$ for rapid degradation by the $26 \mathrm{~S}$ proteasome. Degradation of IKB allows NF- $\mathrm{KB}$

Received for publication April 22, 2003, and accepted in revised form December 2, 2003.

Address correspondence to: Jens C. Brüning, Institute for Genetics, Department of Mouse Genetics and Metabolism, Weyertal 121, 50931 Köln, Germany. Phone: 49-221-470-2467; Fax: 49-221-470-5185; E-mail: jens.bruening@uni-koeln.de. Mathias Röhl and Manolis Pasparakis contributed equally to this work.

Dinesh Gautam's present address is: National Institute of Diabetes and Digestive and Kidney Diseases, NIH, Bethesda, Maryland, USA.

Conflict of interest: The authors have declared that no conflict of interest exists.

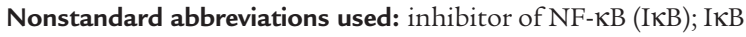
kinase (IKK); insulin receptor substrate 1 (IRS-1); insulin receptor (IR); gold thioglucose (GTG); muscle creatinine kinase (MCK); white adipose tissue (WAT). dimers to translocate to the nucleus and activate transcription of target genes (3).

The IKK complex, which consists of two catalytic subunits, IKK1 and IKK2, and a regulatory subunit, $\mathrm{NEMO} / \mathrm{IKK} \gamma$, is the master regulator of NF-KB-mediated innate immune and inflammatory responses. Of the two catalytic subunits, only IKK2 is essential for $N F-\kappa B$ activation in response to proinflammatory stimuli. Therefore, homozygous IKK2-deficient mouse embryos die at 14.5 days of gestation because of liver degeneration and apoptosis $(4,5)$. IKK2-deficient embryonic fibroblasts have both reduced basal NF- $\kappa B$ activity and impaired cytokine-induced NF- $\kappa B$ activation. Similarly, basal and cytokine-inducible kinase activities of the IKK complex are greatly reduced in IKK2-deficient cells $(4,5)$. These results indicate that IKK2 is crucial for proinflammatory signal-induced activation of NF-KB and that IKK1 can only partially compensate for the loss of IKK2. IKK1 is not essential for NF- $\mathrm{KB}$ activation by proinflammatory stimuli but is important for the inducible processing of p100. In addition, IKK1 plays a critical, apparently NF- $\mathrm{KB}$-independent, role in the differentiation of epidermal keratinocytes (6).

Besides its classical proinflammatory function, TNF- $\alpha$ has been shown to affect metabolism in vivo and adipocytes in vitro. An induction of TNF- $\alpha$ mes- 
senger RNA expression was observed in adipose tissue from different rodent models of obesity and diabetes (7). Neutralization of TNF- $\alpha$ in obese $f a / f a$ rats caused a significant increase in the peripheral uptake of glucose in response to insulin, indicating a role for TNF- $\alpha$ in obesity-induced insulin resistance (7). In vitro, treatment of cultured murine adipocytes with TNF- $\alpha$ induced serine phosphorylation of insulin receptor substrate 1 (IRS-1), thus converting IRS- 1 into an inhibitor of insulin receptor (IR) tyrosine kinase activity (8). In vivo, the absence of TNF- $\alpha$ significantly improved insulin sensitivity in both diet-induced obesity and the $o b / o b$ mouse model. Both TNF- $\alpha$-deficient and TNF- $\alpha$ receptor-deficient obese mice had lower levels of circulating FFAs and were protected from the obesity-related reduction in IR signaling (9). These results indicate that TNF- $\alpha$ is an important mediator of insulin resistance in obesity through its effects on several sites of insulin action.

While both in vitro and in vivo experiments have revealed that TNF- $\alpha$-induced insulin resistance is mediated predominantly through the p55 TNF receptor, the intracellular signaling cascade that leads to this effect remains unclear (9). It has been demonstrated that TNF- $\alpha$-stimulated JNK phosphorylates IRS- 1 at serine residue 307, and that mutation of this serine residue abrogates TNF- $\alpha-$ mediated inhibition of IRS- 1 signaling in cultured cells (10). Accordingly, it has been shown that inactivation of JNK-1 in mice can prevent obesity-induced insulin resistance (11). On the other hand, experimental evidence has been provided that IKK2 can act as the intracellular mediator of TNF- $\alpha$-induced insulin resistance. In cultured fat and hepatoma cells, both salicylate, a characterized inhibitor of IKKs, and expression of a dominant negative mutant IKK2 reversed TNF- $\alpha$-mediated inhibition of IR tyrosine kinase activity (12). Moreover, in vivo heterozygosity for an IKK2-null allele improved insulin sensitivity in the $o b / o b$ obese diabetic mouse model (12).

To further analyze the role of IKK2 in obesityinduced insulin resistance, we have conditionally inactivated the IKK2 gene in mouse skeletal muscle and have analyzed insulin action in lean and obese musclespecific IKK2-deficient mice. We find that, after induction of obesity by injection of gold thioglucose (GTG), the degree of insulin resistance was similar in obese wild-type and muscle-specific IKK2-deficient mice, at both a molecular and a physiological level. Moreover, impaired glucose tolerance resulting from a high-fat diet occurred to the same degree in control and IKK2 mutant mice. Taken together, our data argue against a substantial role for IKK2 action in skeletal muscle in mediating obesity-induced insulin resistance in vivo.

\section{Methods}

Animals and genotyping. For muscle-specific IKK2 disruption, $I K K 2^{f l o x}$ mice were bred with mice expressing Cre recombinase under control of the muscle creati- nine kinase (MCK) promoter $(13,14)$. Animals were housed in a virus-free facility on a 12-hour light/dark cycle and were fed a standard rodent chow. All protocols for animal use and euthanasia were reviewed by the animal care committee of the University of Cologne and were in accordance with NIH guidelines. Genotyping was performed by PCR using genomic DNA isolated from tail tips as described previously $(2,13)$. For obesity induction, animals were injected with $0.5 \mathrm{mg} / \mathrm{g}$ body weight of GTG in $0.9 \%$ saline or with saline alone by the age of 2 weeks or fed a high-fat diet (Altromin, Lage, Germany) from 4 to 20 weeks of age.

Immunoprecipitations and Western blot analysis. Tissues were removed and homogenized in homogenization buffer (50 mM HEPES [pH 7.4], 1\% Triton X-100, 50 $\mathrm{mM}$ sodium pyrophosphate, $0.1 \mathrm{M}$ sodium fluoride, $10 \mathrm{mM}$ EDTA, $10 \mathrm{mM}$ sodium orthovanadate, 10 $\mu \mathrm{g} / \mathrm{ml}$ aprotinin, $10 \mu \mathrm{g} / \mathrm{ml}$ leupeptin, $2 \mathrm{mM}$ benzamidine, and $2 \mathrm{mM}$ PMSF) with a Polytron homogenizer (Ultraturax T25, Jahnke und Kinke, IKALabortechnik, Staufen, Germany). Samples were allowed to solubilize for 30 minutes on ice, and particulate matter was removed by centrifugation at $75,000 \mathrm{~g}$ for 1 hour at $4^{\circ} \mathrm{C}$ in a Sorvall centrifuge (Beckman, Krefeld, Germany). Immunoprecipitations and Western blotting of insulin-signaling proteins were performed as previously described (14).

Northern blot. Total RNA from white adipose tissue (WAT) was prepared using Trizol RNA extraction reagent according to the manufacturer's guidelines. RNA gels, blots, and hybridizations with a radiolabeled TNF- $\alpha$ cDNA probe were performed as previously described (7).

Analytical procedures. Blood glucose values were determined from whole venous blood using an automatic glucose monitor (Glucometer Elite; Bayer, Leverkusen, Germany). Insulin and leptin levels in serum were measured by ELISA using mouse standards according to the manufacturer's guidelines (Crystal Chem Inc., Downers Grove, Illinois, USA). Adiponectin levels in serum were measured by ELISA using mouse standards according to the manufacturer's guidelines (B-Bridge International Inc., San Jose, California, USA). Remaining determinations were performed on a Beckman CX7 analyzer (Beckman).

Glucose-tolerance and insulin-tolerance tests were performed on animals that had been fasted overnight for 16 hours. Animals were injected with either $2 \mathrm{~g} / \mathrm{kg}$ body weight of glucose or $0.75 \mathrm{U} / \mathrm{kg}$ body weight of human regular insulin (Novo Nordisk, Copenhagen, Denmark) into the peritoneal cavity. Glucose levels were determined in blood collected from the tail tip immediately before and 15, 30, 60, and 120 minutes after the injection.

\section{Results}

Efficient muscle-specific IKK2 inactivation. For musclespecific IKK2 inactivation, mice carrying a loxPflanked IKK2 allele were crossed with transgenic mice 


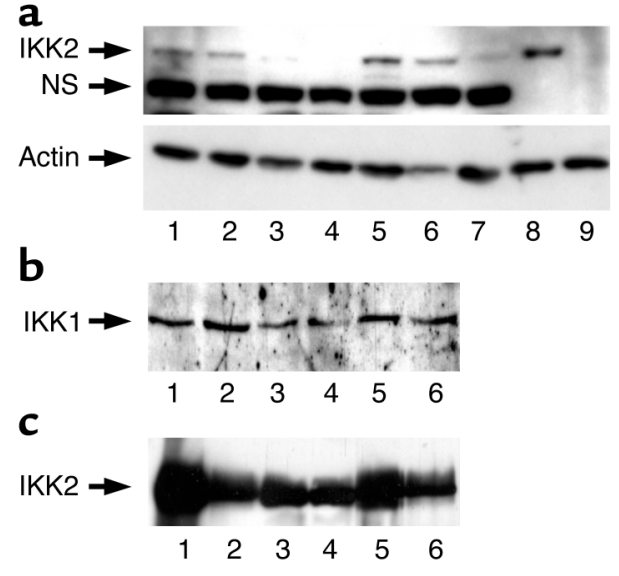

\section{Figure 1}

Efficient muscle-specific deletion of IKK2 in IKK2 mutant mice. (a) The upper panel shows a Western blot analysis of protein extracts prepared from control and conditionally IKK2-deficient mice (lanes 1-7) and from mouse embryonic fibroblasts of control (lane 8) and conventional IKK2-deficient mice (lane 9) probed with an antiIKK2-specific antiserum. The genotypes of the mice are as follows: $I K K 2^{W T}$, lanes 1 and 5 ; IKK $2^{\text {Het }}$, lanes 2 and 6 ; IKK $2^{\text {Mus }}$, lane 3;

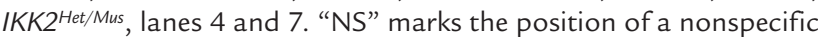
band detected by the antiserum in skeletal muscle. The lower panel shows a Western blot analysis of the same blot shown in the upper panel, after stripping and reprobing with an anti-actin-specific antiserum. (b) Western blot analysis of IKK1 expression in skeletal muscle of different IKK2 mutant mice. The genotypes of the mice are as follows: IKK2 ${ }^{W T}$, lanes 1 and 5 ; IKK $2^{\text {Het }}$, lanes 2 and 6 ; IKK2Mus, lane 3; IKK2 $2_{\text {Het/Mus }}$, lane 4. (c) Western Blot analysis of IKK2 expression in adipose tissue of different conditional IKK2 mutant mice. The genotypes of the mice are as follows: IKK2 ${ }^{W T}$, lanes 1 and $5 ; I K K 2^{\mathrm{Het}}$, lanes 2 and $6 ;$ IKK2 $2^{\text {Mus }}$, lane $3 ;$ IKK $2^{\text {Het/Mus, lane } 4 .}$

analysis with IKK2-specific antibodies. This analysis revealed that the presence of one IKK2-null allele in $I K K 2^{\mathrm{Het}}$ mice resulted in an approximately $50 \%$ reduction of IKK2 expression (Figure 1a). Expression of IKK2 in skeletal muscle of IKK2 $2^{\text {Mus }}$ mice was reduced by more than $90 \%$, as it was in skeletal muscle of $I K K 2^{\mathrm{Het} / \mathrm{Mus}}$ mice (Figure 1a). To control for equal loading of the blots, membranes were stripped and IKK2-deficient (IKK2 $2^{\mathrm{Het}}$ ) mice (15). These animals were then intercrossed with $I K K 2^{\text {flox/flox } M C K C r e}$ mice to obtain four different groups of animals: IKK2 flox/flox (controls), IKK2flox/Het (50\% IKK2 deficiency in all tissues), IKK2 $2^{\text {flox/flox }}$ MCKCre (complete IKK2 deficiency in skeletal muscle), and IKK2 flox/Het MCKCre (50\% IKK2 deficiency in all tissues plus complete IKK2 deficiency in skeletal muscle). For simplicity, the four groups of mice will be referred to as $I K K 2^{W T}$ for controls, $I K K 2^{\text {Het }}$ for mice exhibiting a $50 \%$ reduction throughout the entire body, IKK2Mus for muscle-specific knockouts of IKK2, and IKK2 $2^{\mathrm{Het} / \mathrm{Mus}}$ for mice exhibiting a $50 \%$ reduction throughout the body plus a complete inactivation of the IKK2 gene in skeletal muscle. IKK2 mutant mice were born with the expected mendelian frequency and were morphologically indistinguishable from control mice.

To assess the efficiency of IKK2 inactivation, protein extracts were prepared from skeletal muscle of mice of the different genotypes and subjected to Western blot

\section{Figure 2}

GTG treatment results in obesity. (a) Growth curves of lean male mice (open squares) as compared with GTG-treated male mice (filled squares). Data represent the mean \pm SEM of at least 16 animals in each group. Weights are significantly different between lean and obese mice from week 8 on $(P<0.05)$. (b) The body weight of GTGtreated male mice, according to their IKK2 genotype. Data represent the mean \pm SEM of at least six animals in each group. (c) Plasma leptin concentrations in lean and obese mice, given as mean \pm SEM of at least 16 animals in each group $\left({ }^{*} P<0.01\right)$. (d) Plasma leptin concentrations in GTG-treated male mice of the indicated genotype, given as mean \pm SEM of at least 6 animals in each group, revealed no significant differences depending on IKK2 expression.

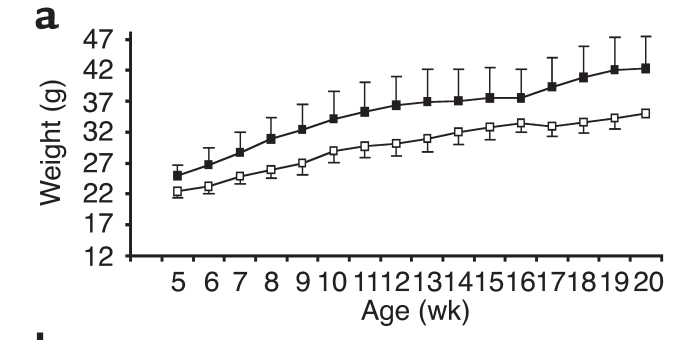

b
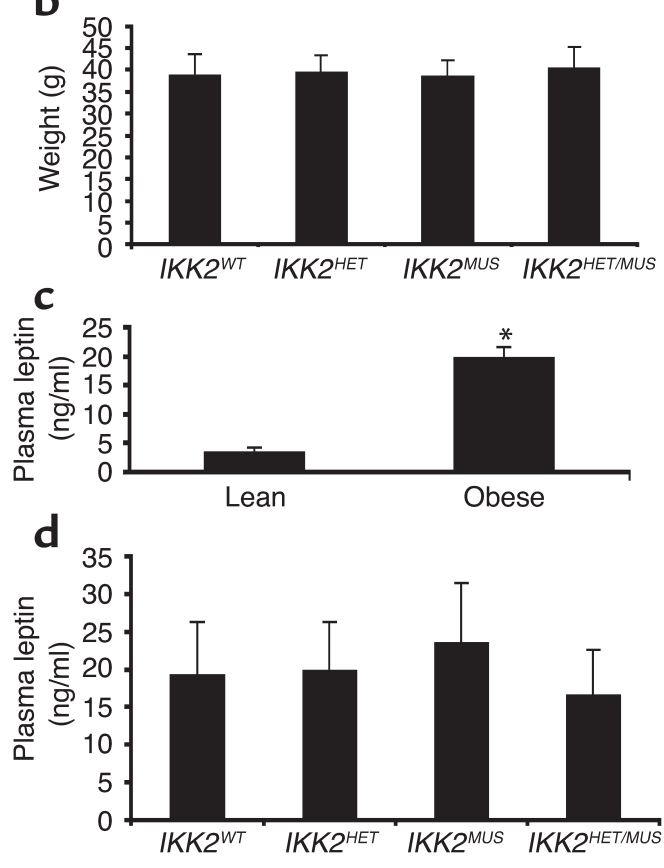
reprobed with an anti-actin antibody, indicating similar protein content of the lysates analyzed (Figure 1a). Moreover, expression of IKK1 remained unaltered in the presence of various degrees of IKK2 inactivation (Figure $1 \mathrm{~b}$ ). To assess the specificity of musclerestricted IKK2 inactivation, IKK2 expression was analyzed by Western blot analysis on extracts prepared from WAT of these mice. As shown in Figure 1c, mice carrying one IKK2-null allele exhibited the expected reduction in IKK2 expression, while the presence of the muscle-restricted MCKCre transgene did not affect IKK2 expression in WAT. Similar results were obtained when IKK2 expression was analyzed in liver of the different mice (data not shown). These data indicate that the targeting strategy outlined above efficiently abolished IKK2 expression in skeletal muscle of the different groups of animals.

IKK2 mutant mice exhibit normal growth rates and develop obesity after GTG treatment. To analyze the role of IKK2 in obesity-induced insulin resistance, we per- formed intraperitoneal injections of either GTG or PBS in 2-week-old mice. When body weight was determined, no difference was detectable between control and IKK2 mutant mice after both PBS and GTG treatment. Both control and IKK2 mutant mice began to gain significantly more weight by the age of 8 weeks than their PBS-treated littermates (Figure 2a). By the age of 16 weeks, GTG-treated mice weighed about $25 \%$ more than their PBS-treated littermates, and no difference was detectable between GTG-treated control and IKK2 mutant mice (Figure 2b). These data indicate that GTG treatment effectively created an obese phenotype in these mice and that skeletal muscle-specific IKK2 disruption or whole-body heterozygosity for an IKK2-null allele had no influence on body weight or the development of obesity.

To further characterize the obese phenotype resulting from GTG treatment, we determined plasma leptin concentrations in the different groups of experimental animals, since previous studies have a

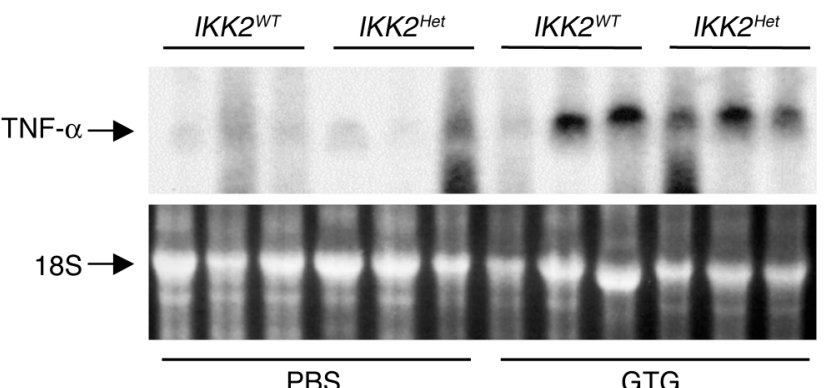

b

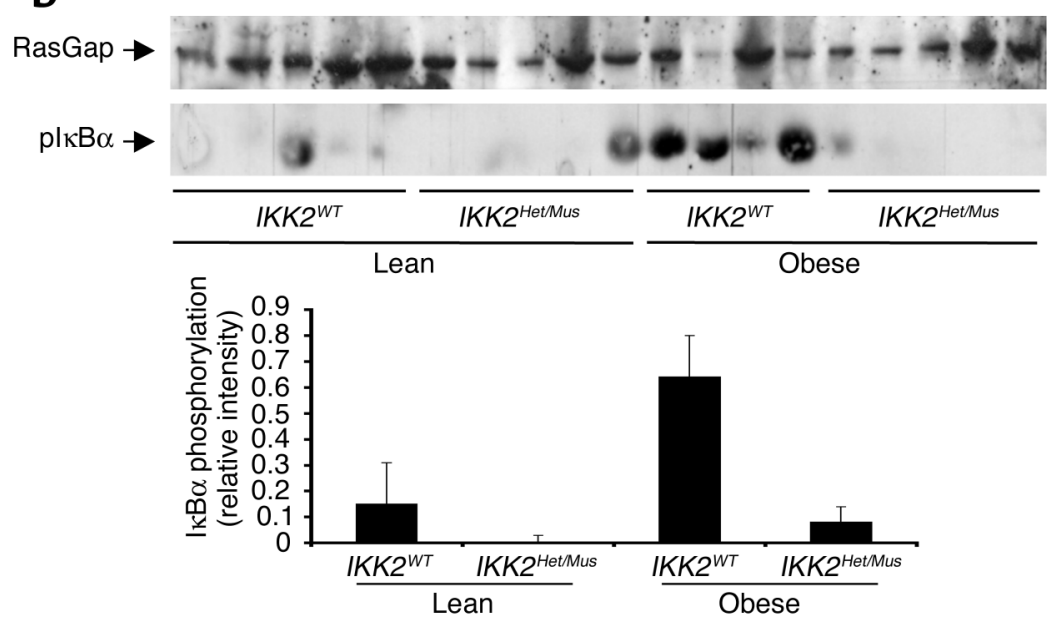

c

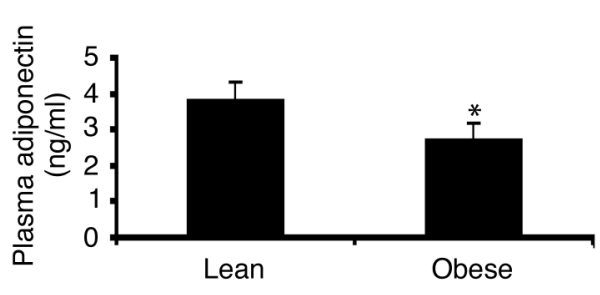

d

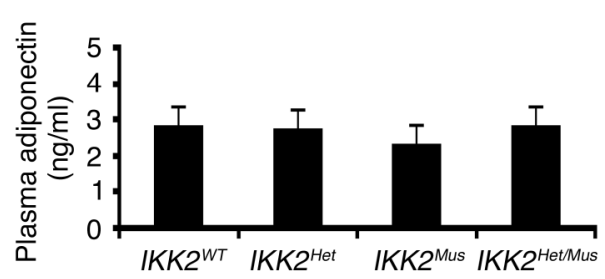

\section{Figure 3}

GTG treatment results in increased TNF- $\alpha$ expression in WAT, activation of TNF- $\alpha$ signaling in skeletal muscle, and decreased plasma adiponectin concentrations. (a) The upper panel shows a Northern blot analysis of RNA extracted from WAT of control and IKK2 Het mice injected either with PBS or GTG. "TNF- $\alpha$ " marks the position of the murine TNF- $\alpha$ mRNA. The lower panel shows a photograph of the ethidium bromide-stained RNA gel prior to transfer onto a nylon membrane as a loading control. "18S" marks the position of the 18S RNA. (b) Protein extracts were prepared from skeletal muscle of lean and obese IKK2 ${ }^{\mathrm{WT}}$ and IKK2 ${ }^{\mathrm{Het} / \text { Mus }}$ mice and subjected to SDS-PAGE followed by Western transfer. After transfer onto nitrocellulose filters, blots were cut and the upper part was subjected to Western blot analysis with an antiserum against RasGap as a loading control, while the lower part was subjected to Western blot analysis with an antiserum that detects $\mathrm{I} \mathrm{KB} \alpha$ when phosphorylated at serine 32 . The lower panel shows the densitometric quantification of I $\mathrm{KB} \alpha \mathrm{phosphorylation}$ as mean $\pm \mathrm{SEM}$ of four to six animals in each group. (c) Plasma adiponectin concentrations in lean and obese mice, given as mean \pm SEM of at least 16 animals in each group $\left({ }^{*} P<0.01\right)$. (d) Plasma adiponectin concentrations in GTG-treated male mice of the indicated genotype, given as mean \pm SEM of at least six animals in each group, revealed no significant differences depending on IKK2 expression. 

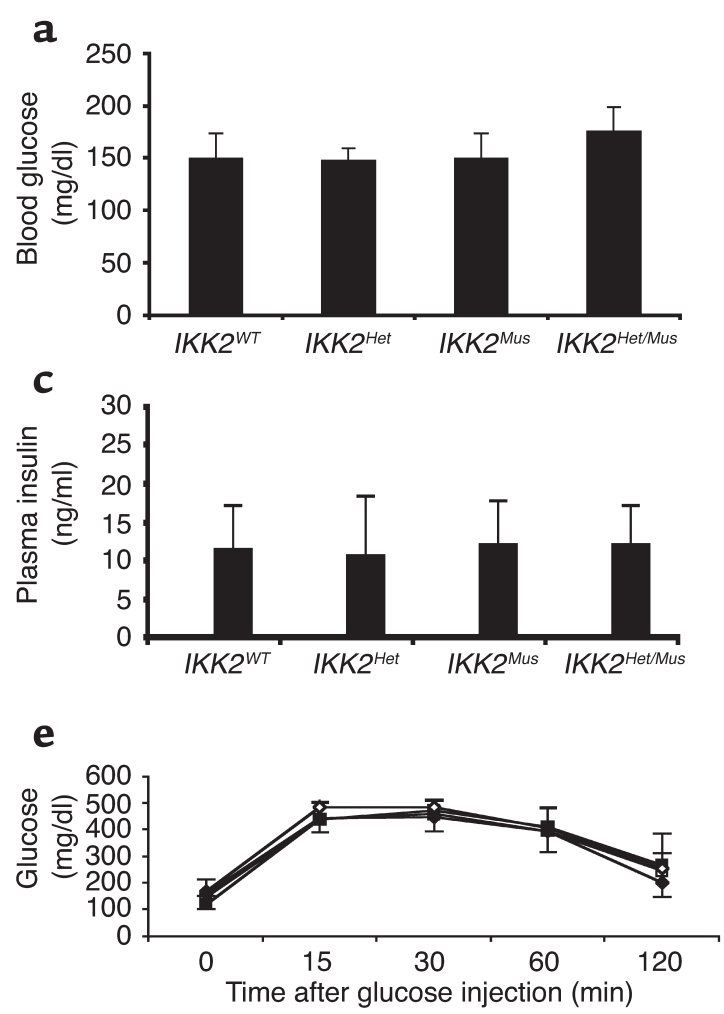

b
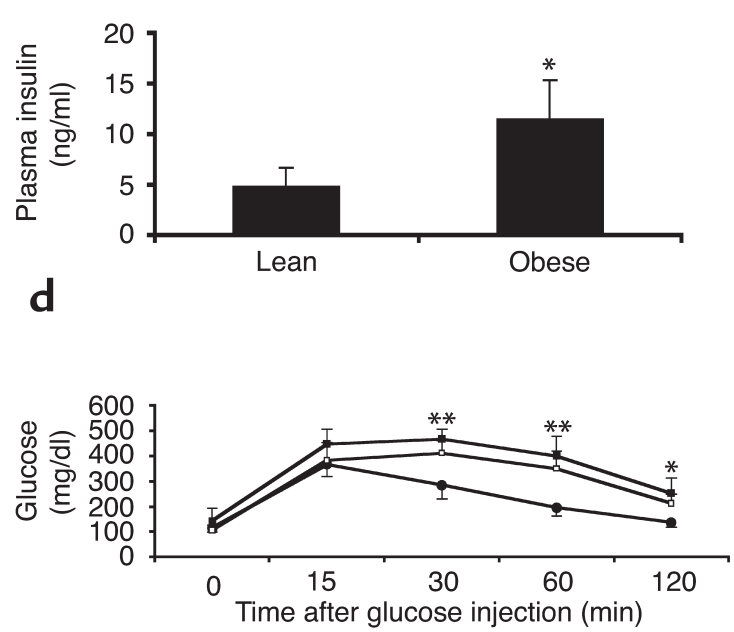

f

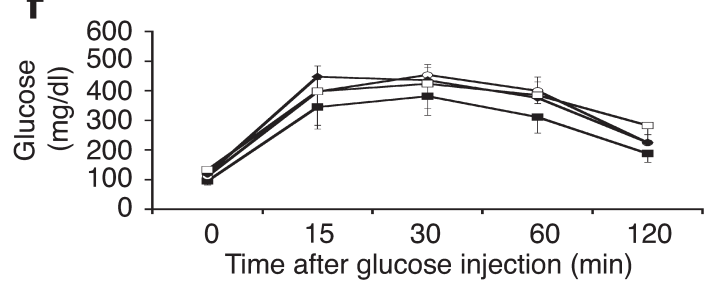

\section{Figure 4}

Glucose metabolism in obese IKK2-deficient mice. (a) Random-fed blood glucose concentrations in male GTG-treated animals of the indicated genotype. Data represent the mean \pm SEM of at least six animals in each group. Statistical analysis revealed no significant differences between the groups (unpaired Student's $t$ test). (b) Plasma insulin concentrations in lean and obese mice, given as mean \pm SEM of at least 16 animals in each group $\left({ }^{*} P<0.05\right)$. (c) Plasma insulin concentrations in male GTG-treated animals of the indicated genotype. Data represent the mean \pm SEM of at least six animals in each group. Statistical analysis revealed no significant differences between the groups (unpaired Student's $t$ test). (d) The results of glucose-tolerance tests in male lean mice (filled squares), GTG-treated mice (open squares), and mice fed a high-fat diet (filled circles). Data represent the mean \pm SEM of at least 12 animals in each group $\left({ }^{*} P<0.05,{ }^{*} P<0.01\right.$, unpaired Student's $t$ test). (e) The results from glucose-tolerance tests in male GTG-treated animals. Data represent the mean \pm SEM of at least six animals in each group (IKK2 ${ }^{W T}$ mice, filled diamonds; IKK2 ${ }^{\text {Het }}$ mice, filled squares; IKK2 ${ }^{\text {Mus }}$ mice, open squares; IKK2 $2^{\text {Het/Mus }}$ mice, open diamonds). (f) The results from glucose-tolerance tests in male animals exposed to a high-fat diet. Data represent the mean \pm SEM of at least six animals in each group (IKK2 ${ }^{W T}$ mice, filled diamonds; IKK2 ${ }^{\text {Het }}$ mice, filled squares; IKK2 ${ }^{\text {Mus }}$ mice, open squares; IKK2 $2^{\text {Het/Mus }}$ mice, open circles).

demonstrated that plasma leptin concentrations correlate with the degree of obesity in a variety of animal models of obesity, including that which results from GTG treatment (16). There was a significant, approximately sixfold increase in circulating plasma leptin concentrations in GTG- versus PBS-treated mice (Figure 2c). Again, there was no significant difference in circulating plasma leptin concentrations between control and IKK2-deficient mice within these groups (Figure 2d). Taken together, GTG-treated animals developed obesity to a similar extent, independent of whole-body heterozygosity for an IKK2-null allele or the presence or absence of IKK2 in skeletal muscle.

Since previous studies have proposed that obesityinduced insulin resistance results from increased TNF- $\alpha$ produced in adipose tissue, we determined TNF- $\alpha$ mRNA expression in WAT of the different mice. TNF- $\alpha$ mRNA was barely detectable in WAT from lean control and IKK2 mutant mice (Figure 3a). By contrast, in the obese animals there was a dramat- ic increase of TNF- $\alpha$ expression in WAT (Figure 3a). No significant difference was detectable between obese control and IKK2 mutant mice (Figure 3a), which demonstrates that obesity resulted in increased TNF- $\alpha$ expression but did so independently of reduced IKK2 expression. To further investigate whether increased TNF- $\alpha$ expression in adipose tissue in response to obesity results in enhanced TNF- $\alpha-$ induced signaling in skeletal muscle, we next determined the amount of phosphorylated IKB $\alpha$ in skeletal muscle of lean and obese mice by Western blot analysis with an antiserum, which recognizes IKB $\alpha$ only in its phosphorylated state. This analysis revealed a significant increase in I $\kappa \mathrm{B} \alpha$ phosphorylation in skeletal muscle of GTG-treated obese control mice as compared with lean controls (Figure 3b). Importantly, this increase was not detectable in obese mice lacking IKK2 expression in skeletal muscle (Figure $3 b)$. These data indicate that GTG treatment results in increased TNF- $\alpha$ expression in WAT and 


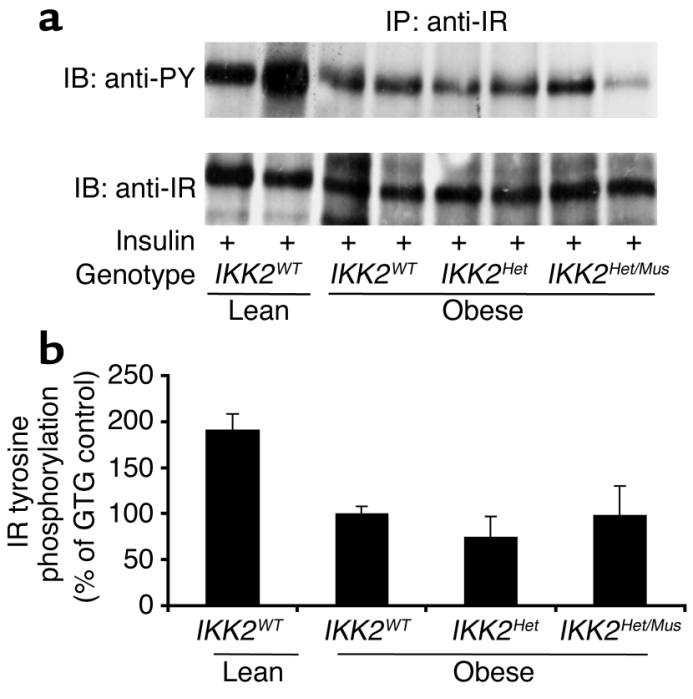

Figure 5

Insulin-stimulated IR activation in skeletal muscle of IKK2-deficient mice. (a) Protein extracts isolated from skeletal muscle of mice, which had been injected with $5 \mathrm{IU}$ of regular insulin 5 minutes before harvesting, were immunoprecipitated with an anti-IR-specific antiserum. The upper panel shows Western blot analysis using an anti-phosphotyrosine-specific antibody (anti-PY). The lower panel shows the autoradiogram after reprobing of the same blot with an anti-IR antiserum. IB, immunoblot; IP, immunoprecipitation. (b) Shown is the densitometric quantification of insulin-stimulated tyrosine phosphorylation of the IR $\beta$ subunit from multiple experiments as outlined in a. The insulin-stimulated IR tyrosine phosphorylation of obese control $\left(I K K 2^{W T}\right)$ mice was arbitrarily set as $100 \%$. The results represent the mean of two to five animals of the indicated genotype. Insulinstimulated IR tyrosine phosphorylation was significantly decreased in obese versus lean animals $(P<0.05)$, but without significant differences between obese animals of the different IKK2 genotypes.

increased activation of the TNF- $\alpha$ signaling cascade in skeletal muscle, resulting in hyperphosphorylation of $I \kappa B \alpha$. Moreover, this effect is abolished in mice lacking IKK2, indicating that IKK2 is responsible for obesity-induced activation of IKB $\alpha$ phosphorylation.

More recently, it was demonstrated that adiponectin represents another factor that is secreted from adipose tissue and regulates systemic insulin sensitivity (17). Therefore, we determined whether obesity resulting from GTG treatment was accompanied by changes in plasma adiponectin concentrations. This analysis revealed a significant decrease in plasma adiponectin concentrations in GTG-treated animals as compared with PBS-injected mice (Figure 3c). Again, there was no significant difference in plasma adiponectin concentrations between obese control and IKK2 mutant mice (Figure 3d). These data indicate that the dysregulation of TNF- $\alpha$ expression and adiponectin secretion following induction of obesity occurred to the same extent regardless of IKK2 deficiency.

Glucose homeostasis is unaltered in muscle-specific IKK2deficient mice. We next determined the impact of IKK2 expression on glucose homeostasis. We first determined blood glucose concentrations in both the fasted and the fed state in the different groups of mice. No difference in fasting or fed blood glucose concentrations was detectable between obese control and IKK2-deficient mice (Figure 4a). Plasma insulin concentrations showed a significant, approximately twofold increase in obese animals as compared with lean mice (Figure $4 \mathrm{~b}$ ), but no significant differences between controls and the different groups of IKK2 mutant mice; this indicated the presence of insulin resistance in both obese control and IKK2 mutant mice (Figure 4c). To further characterize insulin sensitivity in these animals, intraperitoneal insulin-tolerance tests were performed. Obese mice exhibited a blunted response to exogenously administered insulin as compared with lean mice, regardless of their IKK2 genotype (data not shown). Similarly, intraperitoneal glucose-tolerance tests revealed impaired glucose tolerance in the obese mice (Figure $4 d)$. Again there was no difference between obese control mice and the three groups of IKK2 mutant mice (Figure 4e). Taken together, our data show that although GTG administration resulted in obesityassociated insulin resistance and impaired glucose tolerance, there was no difference in insulin sensitivity or glucose tolerance between control and IKK2 mutant mice in vivo. To further elucidate the role of IKK2 in obesity-meditated impairment of glucose homeostasis, another group of animals was placed on a high-fat diet. These mice also exhibited an impairment of glucose tolerance, comparable to that found in GTG-treated mice (Figure 4d). Again, partial deficiency of IKK2 in IKK2 $2^{\text {Het }}$ mice and muscle-specific disruption of IKK2 did not affect the impairment in glucose tolerance as compared with that in control mice receiving the same diet (Figure 4f). These data indicate that disruption of IKK2 signaling in skeletal muscle does not affect the impairment of insulin action that results either from GTG-induced obesity or from high-fat feeding.

Insulin signaling in skeletal muscle of muscle-specific IKK2deficient mice. To characterize obesity-induced insulin resistance in control and IKK2 mutant mice at a molecular level, we determined the expression and insulin-stimulated tyrosine phosphorylation of the IR $\beta$ subunit in skeletal muscle of the different groups of mice, since it has been previously demonstrated that both obesity-induced and TNF- $\alpha$-induced insulin resistance results in decreased insulin-stimulated IR tyrosine phosphorylation and that this defect can be restored by salicylate treatment (17). While expression of the IR $\beta$ subunit was unaltered in obese mice, insulin-stimulated tyrosine phosphorylation was reduced by about $50 \%$ in the obese animals compared with lean controls (Figure 5, a and b). Again, in the obese animals, the reduction of insulin-stimulated IR tyrosine phosphorylation was independent of altered IKK2 expression, indicating that both partial and complete skeletal muscle-specific IKK2 deficiency fails to reverse obesity-induced insulin resistance at 
the level of impaired IR tyrosine phosphorylation (Figure 5, a and b). Similarly, insulin-stimulated activation of the PI3K-dependent PKB/Akt pathway was not different between obese control and IKK2 mutant mice (data not shown).

\section{Discussion}

Although the importance of TNF- $\alpha$ in the development of obesity-associated insulin resistance has been clearly demonstrated over the last decade, the exact molecular mechanisms by which TNF- $\alpha$ induces insulin resistance have still not been fully elucidated. Here, we provide genetic evidence that IKK2 expression in skeletal muscle is not essential for obesityinduced insulin resistance in the models studied. While the data shown in this report were obtained for male GTG-treated mice, amelioration of GTGinduced obesity also appeared to be absent in female mice upon deletion of IKK2 (data not shown). Similarly, insulin resistance resulting from a high-fat diet was not influenced by heterozygosity for an IKK2-null allele and/or muscle-specific IKK2 deletion. This is surprising in view of reports that heterozygosity for an IKK2-null allele can reverse insulin resistance in the $o b / o b$ mouse as well as insulin resistance induced by fat infusion $(12,18)$. Obesity in $o b / o b$ mice is clearly more pronounced than that which occurs after GTG treatment. Therefore, this paradigm of obesity may make the contribution of IKK2 signaling particularly apparent. While the IKK2-null allele generated in the present study has been demonstrated to result in complete lack of IKK2 activity and impaired NF- $\mathrm{KB}$ activation when present in the homozygous state, embryonic fibroblasts heterozygous for this null allele did not show significant reduction in proinflammatory signal-induced NF- $\kappa B$ activity (19). Furthermore, we have also generated a second IKK2-inactivating allele by deleting only exon 7 . This resulted in an inframe mutation, producing a mutant IKK2 protein that lacked amino acids $160-189$, including two serines of the kinase-activation domain, which has been demonstrated to act as a dominant negative mutant in cultured cells $(19,20)$. Also, mice with GTGinduced obesity that are heterozygous for this allele exhibit insulin resistance comparable to that seen in control mice (data not shown). Taken together, we were unable to detect haploinsufficiency for IKK2 in reversing obesity-mediated insulin resistance in the models analyzed herein. Nevertheless, besides the effect of different degrees of obesity and different targeting strategies, differences in genetic background of the animals studied might account for different phenotypes observed.

On the other hand, while heterozygosity for an IKK2-null allele only subtly reversed obesity-induced insulin resistance in the $o b / o b$ mouse, aspirin and salicylate had a more pronounced effect on the amelioration of obesity-induced and lipid-induced insulin resistance (12). Along this line, Jiang et al. recently reported that in cultured cells, salicylate treatment reversed insulin resistance caused by TNF- $\alpha$ or PMA stimulation (21). Interestingly, in this cell line, TNF- $\alpha$ failed to activate IKK2. On the other hand, salicylate inhibited JNK activation, indicating that salicylate can reverse the inhibitory effect of TNF- $\alpha$ on insulin signaling without influencing IKK2 (21). Another very recent study suggests that, in cultured cells, aspirin inhibits multiple serine kinases, including JNK, and that this inhibition is associated with aspirin's ability to reverse TNF- $\alpha$-mediated inhibition of insulin signaling (22). Nevertheless, this study also demonstrated that TNF- $\alpha$-induced inhibitory serine phosphorylation of IRS-1 was reduced in IKK2-deficient embryonic fibroblasts. These data are consistent with the notion, that the effect of aspirin and salicylate in modulating TNF- $\alpha$-mediated insulin resistance targets multiple serine kinases, including IKK2. Given the fact that genetic JNK deficiency also has a profound effect on obesity-induced insulin resistance, these data argue for a more nonspecific effect of salicylate treatment in reversing TNF- $\alpha$-induced insulin resistance in vivo, possibly by targeting JNK activity (11). Taken together, our data argue against a substantial contribution of IKK2 signaling in skeletal muscle to obesity-induced insulin resistance in the models that we analyzed.

It has been shown that TNF- $\alpha$ expression itself is regulated through an NF-kB-dependent pathway (23). Therefore, it is possible that inhibition of IKK2 activity in adipocytes abrogates the obesity-associated increase of TNF- $\alpha$ expression in adipose tissue. The generation of adipose tissue-specific IKK2-deficient mice will provide an excellent tool to further define the role of IKK2 and NF- $\mathrm{KB}$ signaling in adipose tissue in mediating obesity-induced insulin resistance.

\section{Acknowledgments}

We are grateful to G. Schmall for expert secretarial assistance, to C.R. Kahn for MCKCre transgenic mice, and to G. Hotamisligil for the TNF- $\alpha$ cDNA. The study was supported by funds from the Center for Molecular Medicine Cologne (to J.C. Brüning and W. Krone and to K. Rajewsky).

\footnotetext{
1. Karin, M., and Delhase, M. 2000. The I kappa B kinase (IKK) and NF-kappa B: key elements of proinflammatory signalling. Semin. Immunol. 12:85-98.

2. Zandi, E., Rothwarf, D.M., Delhase, M., Hayakawa, M., and Karin, M. 1997. The IkappaB kinase complex (IKK) contains two kinase subunits, IKKalpha and IKKbeta, necessary for IkappaB phosphorylation and NF-kappaB activation. Cell. 91:243-252.

3. Karin, M., and Ben-Neriah, Y. 2000. Phosphorylation meets ubiquitination: the control of NF-אB activity. Annu. Rev. Immunol. 18:621-663.

4. Li, Z.W., et al. 1999. The IKKbeta subunit of IkappaB kinase (IKK) is essential for nuclear factor kappaB activation and prevention of apoptosis. J. Exp. Med. 189:1839-1845.

5. Tanaka, M., et al. 1999. Embryonic lethality, liver degeneration, and impaired NF-kappa B activation in IKK-beta-deficient mice. Immunity. 10:421-429.

6. Hu, Y., et al. 1999. Abnormal morphogenesis but intact IKK activation in mice lacking the IKKalpha subunit of IkappaB kinase. Science. 284:316-320. 7. Hotamisligil, G.S., Shargill, N.S., and Spiegelman, B.M. 1993. Adipose expression of tumor necrosis factor-alpha: direct role in obesity-linked insulin resistance. Science. 259:87-91.
} 
8. Hotamisligil, G.S., et al. 1996. IRS-1-mediated inhibition of insulin receptor tyrosine kinase activity in TNF-alpha- and obesity-induced insulin resistance. Science. 271:665-668.

9. Uysal, K.T., Wiesbrock, S.M., Marino, M.W., and Hotamisligil, G.S. 1997. Protection from obesity-induced insulin resistance in mice lacking TNFalpha function. Nature. 389:610-614.

10. Aguirre, V., Uchida, T., Yenush, L., Davis, R., and White, M.F. 2000. The c-Jun $\mathrm{NH}(2)$-terminal kinase promotes insulin resistance during association with insulin receptor substrate-1 and phosphorylation of Ser(307). J. Biol. Chem. 275:9047-9054.

11. Hirosumi, J., et al. 2002. A central role for JNK in obesity and insulin resistance. Nature. 420:333-336.

12. Yuan, M., et al. 2001. Reversal of obesity- and diet-induced insulin resistance with salicylates or targeted disruption of Ikkbeta. Science. 293:1673-1677.

13. Pasparakis, M., et al. 2002. TNF-mediated inflammatory skin disease in mice with epidermis-specific deletion of IKK2. Nature. 417:861-866.

14. Bruning, J.C., et al. 1998. A muscle-specific insulin receptor knockout exhibits features of the metabolic syndrome of NIDDM without altering glucose tolerance. Mol. Cell. 2:559-569.

15. Schwenk, F., Baron, U., and Rajewsky, K. 1995. A cre-transgenic mouse strain for the ubiquitous deletion of loxP-flanked gene segments including deletion in germ cells. Nucleic Acids Res. 23:5080-5081.

16. Bryson, J.M., et al. 1999. Plasma insulin rise precedes rise in ob mRNA expression and plasma leptin in gold thioglucose-obese mice. Am. J. Physiol. 276:E358-E364.

17. Yamauchi, T., et al. 2001. The fat-derived hormone adiponectin reverses insulin resistance associated with both lipoatrophy and obesity. Nat. Med. 7:941-946.

18. Kim, J.K., et al. 2001. Prevention of fat-induced insulin resistance by salicylate. J. Clin. Invest. 108:437-446. doi:10.1172/JCI200111559.

19. Schmidt-Supprian, M., et al. 2003. Mature T cells depend on signaling through the IKK complex. Immunity. 19:377-389.

20. Pasparakis, M., Schmidt-Supprian, M., and Rajewsky, K. 2002. IkappaB kinase signaling is essential for maintenance of mature B cells. J. Exp. Med. 196:743-752.

21. Jiang, G., Dallas-Yang, Q., Liu, F., Moller, D.E., and Zhang, B.B. 2003. Salicylic acid reverses phorbol 12-myristate-13-acetate (PMA)- and tumor necrosis factor alpha (TNFalpha)-induced insulin receptor substrate 1 (IRS1) serine 307 phosphorylation and insulin resistance in human embryonic kidney 293 (HEK293) cells. J. Biol. Chem. 278:180-186.

22. Gao, Z., Zuberi, A., Quon, M.J., Dong, Z., and Ye, J. 2003. Aspirin inhibits serine phosphorylation of insulin receptor substrate 1 in tumor necrosis factor-treated cells through targeting multiple serine kinases. J. Biol. Chem. 278:24944-24950.

23. Kuprash, D.V., et al. 1999. Similarities and differences between human and murine TNF promoters in their response to lipopolysaccharide. J. Immunol. 162:4045-4052. 